\title{
Modeling and Control of Space Vehicles with Fuel Slosh Dynamics
}

\author{
Mahmut Reyhanoglu \\ Embry-Riddle Aeronautical University
}

USA

\section{Introduction}

In April of 1957, a Jupiter Intermediate Range Ballistic Missile was terminated 90 seconds after launch due to propellant slosh. Ever since the launch of the early high-efficiency rockets, controlling liquid fuel slosh within a launch vehicle has been a major design concern. Moreover, with today's large and complex spacecraft, a substantial mass of fuel is necessary to place them into orbit and to perform orbital maneuvers. The mass of fuel contained in the tanks of a geosynchronous satellite amounts to approximately $40 \%$ of its total mass (Sidi, 1997). When the fuel tanks are only partially filled, large quantities of fuel move inside the tanks under translational and rotational accelerations and generate the fuel slosh dynamics. The traditional treatment of liquid slosh control began with the inclusion of physical barriers, such as baffles and complete compartmentalization, meant to limit the movement of liquid fuel to small amplitudes of high, negligible frequencies. Later, bladders were added to the list of ways to limit these motions. These techniques, although helpful in some cases, do not completely succeed in canceling the sloshing effects. Moreover, these suppression methods involve adding to the spacecraft structural mass, thereby increasing mission cost.

The effects of baffle positions (and quantities) on sloshing frequency have been studied in the literature (Biswal et al., 2004). The mathematical techniques used in these studies are based on the velocity potential function solved using finite-element analysis. Results show that baffles are more effective when near the free-surface of the fluid. In (Venugopal \& Bernstein, 1996), surface pressure control and surface flap actuators have been proposed for controlling slosh in rectangular tanks. The feedback controllers are designed using a Linear-Quadratic-Gaussian (LQG) synthesis. Fluid is assumed to be incompressible, inviscid, and irrotational. Results show a steady-state slosh amplitude lower than the no-actuator case.

The effect of liquid fuel slosh on spinning spacecraft has also been explored in the literature (Hubert, 2003, 2004). Different slosh motion types - surface waves, bulk fluid motion, and vortices - as well as fluid configurations during spinning are defined (Hubert, 2003). The design of control strategies for a launch vehicle with propellant sloshing has been a topic of extensive research in several works (Blackburn \& Vaughan, 1971; Freudenberg \& Morton, 1992; Hubert, 2004). In (Blackburn \& Vaughan, 1971), an advanced linear model of the Saturn V launch vehicle is developed and a linear optimal control law is proposed to control the vehicle. The work in (Freudenberg \& Morton, 1992) studies the problem of robust control of a launch vehicle subject to aerodynamic, flexible, and slosh mode instabilities. 
It has been demonstrated that pendulum and mass-spring models can approximate complicated fluid and structural dynamics; such models have formed the basis for many studies on dynamics and control of space vehicles with fuel slosh (Peterson et al., 1989). There is an extensive body of literature on the interaction of vehicle dynamics and slosh dynamics and their control, but this literature treats only the case of small perturbations to the vehicle dynamics. However, in this chapter the control laws are designed by incorporating the complete nonlinear translational and rotational vehicle dynamics.

For accelerating space vehicles, several thrust vector control design approaches have been developed to suppress the fuel slosh dynamics. These approaches have commonly employed methods of linear control design (Sidi, 1997; Bryson, 1994; Wie, 2008) and adaptive control (Adler et al., 1991). A number of related papers following a similar approach are motivated by robotic systems moving liquid filled containers (Feddema et al., 1997; Grundelius, 2000; Grundelius \& Bernhardsson, 1999; Yano, Toda \& Terashima, 2001; Yano, Higashikawa \& Terashima, 2001; Yano \& Terashima, 2001; Terashima \& Schmidt, 1994). In most of these approaches, suppression of the slosh dynamics inevitably leads to excitation of the transverse vehicle motion through coupling effects; this is a major drawback which has not been adequately addressed in the published literature.

In this chapter, a spacecraft with a partially filled spherical fuel tank is considered, and the lowest frequency slosh mode is included in the dynamic model using pendulum and mass-spring analogies. A complete set of spacecraft control forces and moments is assumed to be available to accomplish planar maneuvers. Aerodynamic effects are ignored here, although they can be easily included in the spacecraft dynamics assuming that they are canceled by the spacecraft controls. It is also assumed that the spacecraft is in a zero gravity environment, but this assumption is for convenience only. These simplifying assumptions render the problem tractable, while still reflecting the important coupling between the unactuated slosh dynamics and the actuated rigid body motion of the spacecraft. The control objective, as is typical for spacecraft orbital maneuvering problems, is to control the translational velocity vector and the attitude of the spacecraft, while attenuating the slosh mode. Subsequently, mathematical models that reflect all of these assumptions are constructed. These problems are interesting examples of underactuated control problems for multibody systems. In particular, the objective is to simultaneously control the rigid body degrees of freedom and the fuel slosh degree of freedom using only controls that act on the rigid body. Control of the unactuated fuel slosh degree of freedom must be achieved through the system coupling. Finally, linear and nonlinear feedback control laws are designed to achieve this control objective.

It is shown that a linear controller, while successful in stabilizing the pitch and slosh dynamics, fails to control the transverse dynamics of a spacecraft. A Lypunov-based nonlinear feedback control law is designed to achieve stabilization of the pitch and transverse dynamics as well as suppression of the slosh mode while the spacecraft accelerates in the axial direction. The results of this chapter are illustrated through simulation examples.

\section{Mathematical model}

In this section, we formulate the dynamics of a spacecraft with a spherical fuel tank and include the lowest frequency slosh mode. We represent the spacecraft as a rigid body (base body) and the sloshing fuel mass as an internal body, and follow the development in our previous work (Cho et al., 2000a) to express the equations of motion in terms of the spacecraft translational velocity vector, the angular velocity, and the internal (shape) coordinate representing the slosh mode. 
To summarize the formulation in (Cho et al., 2000a), let $\mathbf{v} \in \Re^{3}, \boldsymbol{\omega} \in \Re^{3}$, and $\eta \in \Re$ denote the base body translational velocity vector, the base body angular velocity vector, and the internal coordinate, respectively. In these coordinates, the Lagrangian has the form $L=L(\mathbf{v}, \boldsymbol{\omega}, \eta, \dot{\eta})$, which is $S E(3)$-invariant in the sense that it does not depend on the base body position and attitude. The generalized forces and moments on the spacecraft are assumed to consist of control inputs which can be partitioned into two parts: $\tau_{t} \in \Re^{3}$ (typically from thrusters) is the vector of generalized control forces that act on the base body and $\tau_{r} \in \Re^{3}$ (typically from symmetric rotors, reaction wheels, and thrusters) is the vector of generalized control torques that act on the base body. We also assume that the internal dissipative forces are derivable from a Rayleigh dissipation function $\mathrm{R}$. Then, the equations of motion of the spacecraft with internal dynamics are shown to be given by:

$$
\begin{aligned}
& \frac{d}{d t} \frac{\partial L}{\partial \mathbf{v}}+\hat{\omega} \frac{\partial L}{\partial \mathbf{v}}=\tau_{t}, \\
& \frac{d}{d t} \frac{\partial L}{\partial \omega}+\hat{\omega} \frac{\partial L}{\partial \omega}+\hat{\mathbf{v}} \frac{\partial L}{\partial \mathbf{v}}=\tau_{r}, \\
& \frac{d}{d t} \frac{\partial L}{\partial \dot{\eta}}-\frac{\partial L}{\partial \eta}+\frac{\partial R}{\partial \dot{\eta}}=0,
\end{aligned}
$$

where â denotes a $3 \times 3$ skew-symmetric matrix formed from $\mathbf{a}=\left[a_{1}, a_{2}, a_{3}\right]^{T} \in \Re^{3}$ :

$$
\hat{\mathbf{a}}=\left[\begin{array}{ccc}
0 & -a_{3} & a_{2} \\
a_{3} & 0 & -a_{1} \\
-a_{2} & a_{1} & 0
\end{array}\right]
$$

Note that equations (1)-(2) are identical to Kirchhoff's equations (Meirovitch \& Kwak, 1989), which can also be expressed in the form of Euler-Poincaré equations. It must be pointed out that in the above formulation it is assumed that no control forces or torques exist that directly control the internal dynamics. The objective is to simultaneously control the rigid body dynamics and the internal dynamics using only control effectors that act on the rigid body; the control of internal dynamics must be achieved through the system coupling. In this regard, equations (1)-(3) model interesting examples of underactuated mechanical systems. In our previous research (Reyhanoglu et al., 1996, 1999), we have developed theoretical controllability and stabilizability results for a large class of underactuated mechanical systems using tools from nonlinear control theory. We have also developed effective nonlinear control design methodologies (Reyhanoglu et al., 2000) that we applied to several examples of underactuated mechanical systems, including underactuated space vehicles (Reyhanoglu, 2003; Cho et al., 2000b).

\subsection{Pendulum analogy}

\subsubsection{Spacecraft with throttable side thrusters}

The formulation using a pendulum analogy can be summarized as follows. Consider a rigid spacecraft moving in a fixed plane as indicated in Fig. 1. The important variables are the axial and transverse components of the velocity of the center of the fuel tank, $v_{x}, v_{z}$, the attitude angle $\theta$ of the spacecraft with respect to a fixed reference, and the angle $\psi$ of the pendulum with respect to the spacecraft longitudinal axis, representing the fuel slosh. A thrust $F$, which is assumed to act through the spacecraft center of mass along the spacecraft's longitudinal axis, a transverse force $f$, and a pitching moment $M$ are available for control purposes. The constants in the problem are the spacecraft mass $m$ and moment of inertia $I$ (without fuel), the 


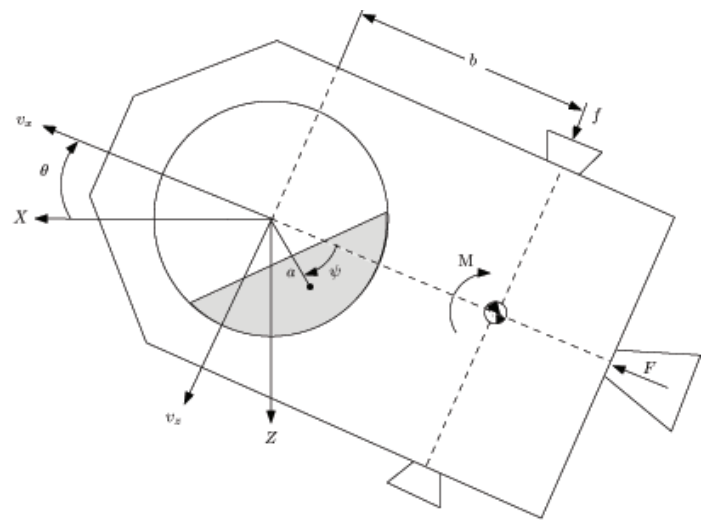

Fig. 1. A single slosh pendulum model for a spacecraft with throttable side thrusters.

fuel mass $m_{f}$ and moment of inertia $I_{f}$ (assumed constant), the length $a>0$ of the pendulum, and the distance $b$ between the pendulum point of attachment and the spacecraft center of mass location along the longitudinal axis; if the pendulum point of attachment is in front of the spacecraft center of mass then $b>0$. The parameters $m_{f}, I_{f}$ and $a$ depend on the shape of the fuel tank, the characteristics of the fuel and the fill ratio of the fuel tank.

Let $\hat{i}$ and $\hat{k}$ denote unit vectors along the spacecraft-fixed longitudinal and transverse axes, respectively, and denote by $(x, z)$ the inertial position of the center of the fuel tank. The position vector of the center of mass of the vehicle can then be expressed in the spacecraft-fixed coordinate frame as

$$
\vec{r}=(x-b) \hat{i}+z \hat{k} .
$$

Clearly, the inertial velocity of the vehicle can be computed as

$$
\begin{aligned}
\dot{\vec{r}} & =(\dot{x}+z \dot{\theta}) \hat{i}+(\dot{z}-x \dot{\theta}+b \dot{\theta}) \hat{k} \\
& =v_{x} \hat{i}+\left(v_{z}+b \dot{\theta}\right) \hat{k},
\end{aligned}
$$

where we have used the fact that $v_{x}=\dot{x}+z \dot{\theta}$ and $v_{z}=\dot{z}-x \dot{\theta}$.

Similarly, the position vector of the center of mass of the fuel lump in the spacecraft-fixed coordinate frame is given by

$$
\vec{r}_{f}=(x-a \cos \psi) \hat{i}+(z+a \sin \psi) \hat{k},
$$

and the inertial velocity of the fuel lump can be computed as

$$
\begin{aligned}
\dot{\vec{r}}_{f} & =[\dot{x}+a \dot{\psi} \sin \psi+\dot{\theta}(z+a \sin \psi)] \hat{i}+[\dot{z}+a \dot{\psi} \cos \psi-\dot{\theta}(x-a \cos \psi)] \hat{k} \\
& =\left[v_{x}+a(\dot{\theta}+\dot{\psi}) \sin \psi\right] \hat{i}+\left[v_{z}+a(\dot{\theta}+\dot{\psi}) \cos \psi\right] \hat{k} .
\end{aligned}
$$

The total kinetic energy can now be expressed as

$$
\begin{aligned}
T & =\frac{1}{2} m \dot{\vec{r}}^{2}+\frac{1}{2} m_{f} \dot{\vec{r}}_{f}^{2}+\frac{1}{2} I \dot{\theta}^{2}+\frac{1}{2} I_{f}(\dot{\theta}+\dot{\psi})^{2} \\
& =\frac{1}{2} m\left[v_{x}^{2}+\left(v_{z}+b \dot{\theta}\right)^{2}\right]+\frac{1}{2} m_{f}\left[\left(v_{x}+a(\dot{\theta}+\dot{\psi}) \sin \psi\right)^{2}+\left(v_{z}+a(\dot{\theta}+\dot{\psi}) \cos \psi\right)^{2}\right] \\
& +\frac{1}{2} I \dot{\theta}^{2}+\frac{1}{2} I_{f}(\dot{\theta}+\dot{\psi})^{2} .
\end{aligned}
$$




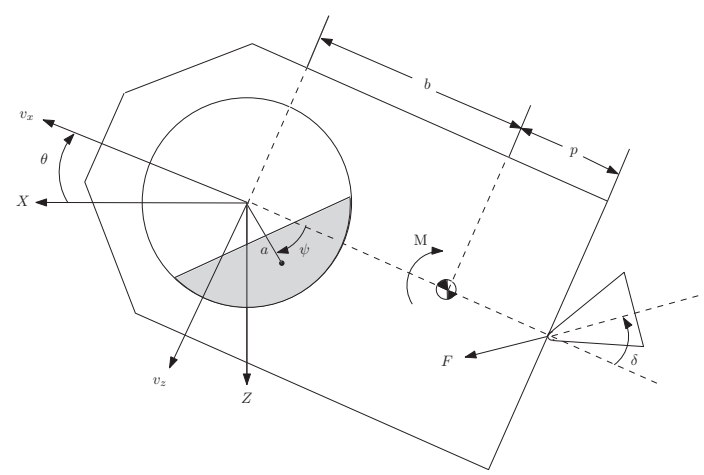

Fig. 2. A single slosh pendulum model for a spacecraft with a gimballed thrust engine.

Since gravitational effects are ignored, there is no potential energy. Thus, the Lagrangian equals the kinetic energy, i.e. $L=T$. Applying equations (1)-(3) with

$$
\eta=\psi, R=\frac{1}{2} \epsilon \dot{\psi}^{2}, \mathbf{v}=\left[\begin{array}{c}
v_{x} \\
0 \\
v_{z}
\end{array}\right], \boldsymbol{\omega}=\left[\begin{array}{l}
0 \\
\dot{\theta} \\
0
\end{array}\right], \boldsymbol{\tau}_{t}=\left[\begin{array}{c}
F \\
0 \\
f
\end{array}\right], \boldsymbol{\tau}_{r}=\left[\begin{array}{c}
0 \\
M+f b \\
0
\end{array}\right]
$$

and rearranging, the equations of motion can be obtained as:

$$
\begin{aligned}
& \left(m+m_{f}\right)\left(\dot{v}_{x}+\dot{\theta} v_{z}\right)+m_{f} a(\ddot{\theta}+\ddot{\psi}) \sin \psi+m b \dot{\theta}^{2}+m_{f} a(\dot{\theta}+\dot{\psi})^{2} \cos \psi=F, \\
& \left(m+m_{f}\right)\left(\dot{v}_{z}-\dot{\theta} v_{x}\right)+m_{f} a(\ddot{\theta}+\ddot{\psi}) \cos \psi+m b \ddot{\theta}-m_{f} a(\dot{\theta}+\dot{\psi})^{2} \sin \psi=f, \\
& \left(I+m b^{2}\right) \ddot{\theta}+m b\left(\dot{v}_{z}-\dot{\theta} v_{x}\right)-\epsilon \dot{\psi}=M+b f, \\
& \left(I_{f}+m_{f} a^{2}\right)(\ddot{\theta}+\ddot{\psi})+m_{f} a\left[\left(\dot{v}_{x}+\dot{\theta} v_{z}\right) \sin \psi+\left(\dot{v}_{z}-\dot{\theta} v_{x}\right) \cos \psi\right]+\epsilon \dot{\psi}=0 .
\end{aligned}
$$

\subsubsection{Spacecraft with a gimballed thrust engine}

Now consider the single slosh pendulum model for a spacecraft with a gimballed thrust engine as shown in Fig. 2, where $\delta$ denotes the gimbal deflection angle, which is considered as one of the control inputs. It is clear that, as in the previous case, the total kinetic energy is given by equation (6) and the Lagrangian equals the kinetic energy. Applying equations (1)-(3) with

$\eta=\psi, R=\frac{1}{2} \epsilon \dot{\psi}^{2}, \mathbf{v}=\left[\begin{array}{c}v_{x} \\ 0 \\ v_{z}\end{array}\right], \boldsymbol{\omega}=\left[\begin{array}{l}0 \\ \dot{\theta} \\ 0\end{array}\right], \boldsymbol{\tau}_{t}=\left[\begin{array}{c}F \cos \delta \\ 0 \\ F \sin \delta\end{array}\right], \boldsymbol{\tau}_{r}=\left[\begin{array}{c}0 \\ M+F(b+p) \sin \delta \\ 0\end{array}\right]$,

and rearranging, the equations of motion can be obtained as:

$$
\begin{aligned}
& \left(m+m_{f}\right)\left(\dot{v}_{x}+\dot{\theta} v_{z}\right)+m_{f} a(\ddot{\theta}+\ddot{\psi}) \sin \psi+m b \dot{\theta}^{2}+m_{f} a(\dot{\theta}+\dot{\psi})^{2} \cos \psi=F \cos \delta, \\
& \left(m+m_{f}\right)\left(\dot{v}_{z}-\dot{\theta} v_{x}\right)+m_{f} a(\ddot{\theta}+\ddot{\psi}) \cos \psi+m b \ddot{\theta}-m_{f} a(\dot{\theta}+\dot{\psi})^{2} \sin \psi=F \sin \delta, \\
& \left(I+m b^{2}\right) \ddot{\theta}+m b\left(\dot{v}_{z}-\dot{\theta} v_{x}\right)-\epsilon \dot{\psi}=M+F(b+p) \sin \delta, \\
& \left(I_{f}+m_{f} a^{2}\right)(\ddot{\theta}+\ddot{\psi})+m_{f} a\left[\left(\dot{v}_{x}+\dot{\theta} v_{z}\right) \sin \psi+\left(\dot{v}_{z}-\dot{\theta} v_{x}\right) \cos \psi\right]+\epsilon \dot{\psi}=0 .
\end{aligned}
$$




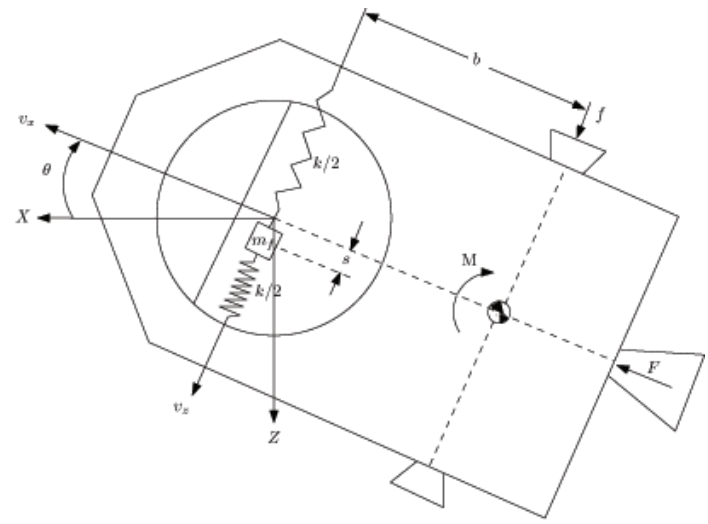

Fig. 3. A single slosh mass-spring model for a spacecraft with throttable side thrusters.

The control objective is to design feedback controllers so that the controlled spacecraft accomplishes a given planar maneuver, that is a change in the translational velocity vector and the attitude of the spacecraft, while attenuating the slosh mode. The work in (Cho et al., $2000 \mathrm{~b}$ ) considers a constant thrust $F>0$ and develops a feedback law (using a backstepping approach) to stabilize the system (7)-(10) to a relative equilibrium defined by a constant acceleration in the axial direction. A slightly modified and relatively simpler feedback controller that uses a Lyapunov approach (without resorting to backstepping) can be found in (Reyhanoglu, 2003). In the subsequent development, we will develop feedback controllers to achieve the same control objective using the system (11)-(14).

\subsection{Mass-spring analogy}

The mass-spring analogy is related to the pendulum analogy, in which the oscillation frequency of the mass-spring element represents the lowest frequency sloshing mode (Sidi, 1997).

Consider a rigid spacecraft moving on a plane as indicated in Fig. 3, where $v_{x}, v_{z}$ are the axial and transverse components, respectively, of the velocity of the center of the fuel tank, and $\theta$ denotes the attitude angle of the spacecraft with respect to a fixed reference. The slosh mode is modeled by a point mass $m_{f}$ whose relative position along the body $z$-axis is denoted by $s$; a restoring force $-k s$ acts on the mass whenever the mass is displaced from its neutral position $s=0$. As in the previous model, a thrust $F$, which is assumed to act through the spacecraft center of mass along the spacecraft's longitudinal axis, a transverse force $f$, and a pitching moment $M$ are available for control purposes. The constants in the problem are the spacecraft mass $m$ and moment of inertia $I$, the fuel mass $m_{f}$, and the distance $b$ between the body $z$-axis and the spacecraft center of mass location along the longitudinal axis. The parameters $m_{f}, k$ and $b$ depend on the shape of the fuel tank, the characteristics of the fuel and the fill ratio of the fuel tank.

The position vector of the fuel mass $m_{f}$ in the spacecraft-fixed coordinate frame is given by

$$
\vec{r}_{f}=x \hat{i}+(z+s) \hat{k}
$$


and the inertial velocity of the fuel can be computed as

$$
\begin{aligned}
\dot{\vec{r}}_{f} & =[\dot{x}+(z+s) \dot{\theta}] \hat{i}+[\dot{z}-x \dot{\theta}+\dot{s}] \hat{k} \\
& =\left(v_{x}+s \dot{\theta}\right) \hat{i}+\left(v_{z}+\dot{s}\right) \hat{k} .
\end{aligned}
$$

Thus, under the indicated assumptions, the Lagrangian can be found as

$$
\begin{aligned}
L & =\frac{1}{2} m \dot{\vec{r}}^{2}+\frac{1}{2} m_{f} \dot{\vec{r}}_{f}^{2}+\frac{1}{2} I \dot{\theta}^{2}-\frac{1}{2} k s^{2} \\
& =\frac{1}{2} m\left[v_{x}^{2}+\left(v_{z}+b \dot{\theta}\right)^{2}\right]+\frac{1}{2} m_{f}\left[\left(v_{x}+s \dot{\theta}\right)^{2}+\left(v_{z}+\dot{s}\right)^{2}\right]+\frac{1}{2} I \dot{\theta}^{2}-\frac{1}{2} k s^{2} .
\end{aligned}
$$

Applying equations (1)-(3) with

$$
\eta=s, R=\frac{1}{2} c \dot{s}^{2}, \mathbf{v}=\left(\begin{array}{c}
v_{x} \\
0 \\
v_{z}
\end{array}\right), \boldsymbol{\omega}=\left(\begin{array}{c}
0 \\
\dot{\theta} \\
0
\end{array}\right), \boldsymbol{\tau}_{t}=\left(\begin{array}{c}
F \\
0 \\
f
\end{array}\right), \boldsymbol{\tau}_{r}=\left(\begin{array}{c}
0 \\
M+f b \\
0
\end{array}\right),
$$

the equations of motion can be obtained as

$$
\begin{aligned}
& \left(m+m_{f}\right)\left(\dot{v}_{x}+\dot{\theta} v_{z}\right)+m_{f} s \ddot{\theta}+m b \dot{\theta}^{2}+2 m_{f} \dot{s} \dot{\theta}=F, \\
& \left(m+m_{f}\right)\left(\dot{v}_{z}-\dot{\theta} v_{x}\right)+m b \ddot{\theta}+m_{f} \ddot{s}-m_{f} s \dot{\theta}^{2}=f, \\
& \left(I+m b^{2}+m_{f} s^{2}\right) \ddot{\theta}+m_{f} s\left(\dot{v}_{x}+\dot{\theta} v_{z}\right)+2 m_{f} s \dot{s} \dot{\theta}+m b\left(\dot{v}_{z}-\dot{\theta} v_{x}\right)=M+b f, \\
& m_{f}\left(\ddot{s}+\dot{v}_{z}-\dot{\theta} v_{x}-s \dot{\theta}^{2}\right)+k s+c \dot{s}=0 .
\end{aligned}
$$

The control objective is again to design feedback controllers so that the controlled spacecraft accomplishes a given planar maneuver, that is a change in the translational velocity vector and the attitude of the spacecraft, while suppressing the slosh mode.

\section{Feedback control design}

In this section, we restrict the development to the single slosh pendulum model of a spacecraft with a gimballed thrust engine, i.e. we design feedback controllers for the system (11)-(14) only. In particular, we study the problem of controlling the system to a relative equilibrium defined by a constant acceleration in the axial direction.

\subsection{Linearization-based controller design}

To obtain the linearized equations of motion, assume small gimbal deflection so that $\cos \delta \approx 1$ and $\sin \delta \approx \delta$, and we rewrite (11)-(14) as:

$$
\begin{aligned}
& \left(m+m_{f}\right) a_{x}+m_{f} a(\ddot{\theta}+\ddot{\psi}) \sin \psi+m b \dot{\theta}^{2}+m_{f} a(\dot{\theta}+\dot{\psi})^{2} \cos \psi=F, \\
& \left(m+m_{f}\right) a_{z}+m_{f} a(\ddot{\theta}+\ddot{\psi}) \cos \psi+m b \ddot{\theta}-m_{f} a(\dot{\theta}+\dot{\psi})^{2} \sin \psi=F \delta, \\
& \left(I+m b^{2}\right) \ddot{\theta}+m b a_{z}-\epsilon \dot{\psi}=M+F l \delta, \\
& \left(I_{f}+m_{f} a^{2}\right)(\ddot{\theta}+\ddot{\psi})+m_{f} a\left(a_{x} \sin \psi+a_{z} \cos \psi\right)+\epsilon \dot{\psi}=0,
\end{aligned}
$$

where $l=b+p$ and $\left(a_{x}, a_{z}\right)=\left(\dot{v}_{x}+\dot{\theta} v_{z}, \dot{v}_{z}-\dot{\theta} v_{x}\right)$ are the axial and transverse components of the acceleration of the center of the fuel tank. The number of equations of motion can 
be reduced to two by solving equations (21) and (22) for $a_{x}$ and $a_{z}$, and eliminating these accelerations from equations (23) and (24).

$$
\begin{aligned}
& {\left[I+m^{*}\left(b^{2}-a b \cos \psi\right)\right] \ddot{\theta}-m^{*} a b \ddot{\psi} \cos \psi+m^{*} a b(\dot{\theta}+\dot{\psi})^{2} \sin \psi-\epsilon \dot{\psi}=M+b^{*} F \delta,} \\
& {\left[I_{f}+m^{*}\left(a^{2}-a b \cos \psi\right)\right] \ddot{\theta}+\left(I_{f}+m^{*} a^{2}\right) \ddot{\psi}+\left(a^{*} F-m^{*} a b \dot{\theta}^{2}\right) \sin \psi+\epsilon \dot{\psi}=-a^{*} F \delta \cos \psi,}
\end{aligned}
$$

where

$$
m^{*}=\frac{m m_{f}}{m+m_{f}}, \quad a^{*}=\frac{m_{f} a}{m+m_{f}}, \quad b^{*}=\frac{m_{f} b}{m+m_{f}}+d .
$$

As mentioned previously, without loss of generality, we will assume that the desired equilibrium is given by:

$$
\left(\theta^{*}, \dot{\theta}^{*}, \psi^{*}, \dot{\psi}^{*}\right)=(0,0,0,0) .
$$

Assuming that $\theta, \dot{\theta}, \psi$, and $\dot{\psi}$ are small, the following linearized equations can be obtained:

$$
\begin{aligned}
& I_{1} \ddot{\theta}-I_{2} \ddot{\psi}-\epsilon \dot{\psi}=M+b^{*} F \delta, \\
& I_{3} \ddot{\theta}+I_{4} \ddot{\psi}+a^{*} F \psi+\epsilon \dot{\psi}=-a^{*} F \delta .
\end{aligned}
$$

where

$$
\begin{aligned}
& I_{1}=I+m^{*}\left(b^{2}-a b\right), I_{2}=m^{*} a b, \\
& I_{3}=I_{f}+m^{*}\left(a^{2}-a b\right), I_{4}=I_{f}+m^{*} a^{2} .
\end{aligned}
$$

For the linearized system (27)-(28), the state variables are the attitude angle $\theta$, the slosh angle $\psi$, and their time derivatives. The collection of these state variables is defined as the partial state vector given by

$$
\mathbf{x}=[\theta, \dot{\theta}, \psi, \dot{\psi}]^{T} .
$$

Let $\mathbf{u}=[\delta, M]^{T}$ denote the control input vector. Then, the state space equations can be written as:

$$
\dot{\mathbf{x}}=\mathbf{A x}+\mathbf{B u},
$$

where

$$
\mathbf{A}=\left[\begin{array}{cccc}
0 & 1 & 0 & 0 \\
0 & 0 & -\frac{a^{*} I_{2} F}{\Delta} & -\frac{\left(I_{2}-I_{4}\right) \epsilon}{\Delta} \\
0 & 0 & 0 & 1 \\
0 & 0 & -\frac{a^{*} I_{1} F}{\Delta} & -\frac{\left(I_{1}+I_{3}\right) \epsilon}{\Delta}
\end{array}\right], \mathbf{B}=\left[\begin{array}{cc}
0 & 0 \\
-\frac{\left(I_{2} a^{*}-I_{4} b^{*}\right) F}{\Delta} & \frac{I_{4}}{\Delta} \\
0 & 0 \\
-\frac{\left(I_{1} a^{*}+I_{3} b^{*}\right) F}{\Delta} & -\frac{I_{3}}{\Delta}
\end{array}\right]
$$

and

$$
\Delta=I_{1} I_{4}+I_{2} I_{3} .
$$

We consider an LQR (Linear Quadratic Regulator) controller of the form

$$
\mathbf{u}=-\mathbf{K x}
$$

that minimizes the quadratic cost function

$$
J=\int_{0}^{\infty}\left(\mathbf{x}^{T} \mathbf{Q} \mathbf{x}+\mathbf{u}^{T} \mathbf{R} \mathbf{u}\right) d t
$$


where $\mathbf{Q}$ is a symmetric positive-semidefinite weighting matrix and $\mathbf{R}$ is a positive-definite weighting matrix.

The optimal control gain matrix $\mathbf{K}$ is found by solving the corresponding matrix Riccati equation (or using MATLAB's lqr function). This controller is then applied to the actual nonlinear system (11)-(14). The simulation results show that the linear controller (31) results in undesirable steady-state errors in transverse velocity (see Figure 4).

\subsection{Lyapunov-based controller design}

Consider the single slosh pendulum model of a spacecraft with a gimballed thrust engine shown in Fig. 2. If the thrust $F$ is a positive constant, and if the gimbal deflection angle and pitching moment are zero, $\delta=M=0$, then the spacecraft and fuel slosh dynamics have a relative equilibrium defined by

$$
\begin{gathered}
v_{x}^{*}(t)=\frac{F}{m+m_{f}} t+v_{x 0}, v_{z}=v_{z}^{*}, \\
\theta=\theta^{*}, \dot{\theta}=0, \psi=0, \dot{\psi}=0,
\end{gathered}
$$

where $v_{z}^{*}$ and $\theta^{*}$ are arbitrary constants, and $v_{x 0}$ is the initial axial velocity of the spacecraft. Note that $\theta^{*}$ and $v_{z}^{*}$ are the desired attitude angle and transverse velocity, chosen as zero here. Now assume the axial acceleration term $a_{x}$ is not significantly affected by small gimbal deflections, pitch changes and fuel motion (an assumption verified in simulations). Consequently, equation (11) becomes:

$$
\dot{v}_{x}+\dot{\theta} v_{z}=\frac{F}{m+m_{f}} .
$$

Substituting this approximation leads to the following equations of motion for the transverse, pitch and slosh dynamics:

$$
\begin{array}{r}
\left(m+m_{f}\right)\left(\dot{v}_{z}-\dot{\theta} v_{x}(t)\right)+m_{f} a(\ddot{\theta}+\ddot{\psi}) \cos \psi+m b \ddot{\theta}-m_{f} a(\dot{\theta}+\dot{\psi})^{2} \sin \psi=F \delta, \\
\left(I+m b^{2}\right) \ddot{\theta}+m b\left(\dot{v}_{z}-\dot{\theta} v_{x}(t)\right)-\epsilon \dot{\psi}=M+F l \delta, \\
\left(I_{f}+m_{f} a^{2}\right)(\ddot{\theta}+\ddot{\psi})+m_{f} a \frac{F}{m+m_{f}} \sin \psi+m_{f} a\left(\dot{v}_{z}-\dot{\theta} v_{x}(t)\right) \cos \psi+\epsilon \dot{\psi}=0,
\end{array}
$$

where $v_{x}(t)$ is considered as an exogenous input.

Define the error variable

$$
\tilde{v}_{x}=v_{x}(t)-v_{x}^{*}(t) .
$$

Then, the equations of motion can be written in the following form

$$
\begin{aligned}
\dot{\tilde{v}}_{x} & =-\dot{\theta} v_{z}, \\
\dot{v}_{z} & =u_{1}+\dot{\theta}\left(\tilde{v}_{x}+v_{x}^{*}(t)\right), \\
\ddot{\theta} & =u_{2}, \\
\ddot{\psi} & =-u_{1} c \cos \psi-u_{2}-d \sin \psi-e \dot{\psi},
\end{aligned}
$$

where

$$
c=\frac{m_{f} a}{I_{f}+m_{f} a^{2}}, d=\frac{F c}{m+m_{f}}, e=\frac{\epsilon}{I_{f}+m_{f} a^{2}}
$$


and $\left(u_{1}, u_{2}\right)$ are new control inputs defined as

$$
\left[\begin{array}{l}
u_{1} \\
u_{2}
\end{array}\right]=\mathbf{M}^{-1}(\psi)\left[\begin{array}{c}
F \sin \delta-m_{f} a \ddot{\psi} \cos \psi+m_{f} a(\dot{\theta}+\dot{\psi})^{2} \sin \psi \\
M+F l \sin \delta+\epsilon \dot{\psi}
\end{array}\right]
$$

where

$$
\mathbf{M}(\psi)=\left[\begin{array}{cc}
m_{f} a \cos \psi+m b & m+m_{f} \\
I+m b^{2} & m b
\end{array}\right] .
$$

Now, we consider the following candidate Lyapunov function for the system (37)-(40):

$$
V=\frac{r_{1}}{2}\left(\tilde{v}_{x}^{2}+v_{z}^{2}\right)+\frac{r_{2}}{2} \theta^{2}+\frac{r_{3}}{2} \dot{\theta}^{2}+r_{4} d(1-\cos \psi)+\frac{r_{4}}{2}(\dot{\theta}+\dot{\psi})^{2},
$$

where $r_{1}, r_{2}, r_{3}$, and $r_{4}$ are positive constants. The function $V$ is positive definite in the domain

$$
D=\left\{\left(\tilde{v}_{x}, v_{z}, \theta, \dot{\theta}, \psi, \dot{\psi}\right) \mid-\pi<\psi<\pi\right\} .
$$

The time derivative of $V$ along the trajectories of (37)-(40) is

$$
\begin{aligned}
\dot{V}= & {\left[r_{1} v_{z}-r_{4} c(\dot{\theta}+\dot{\psi}) \cos \psi\right] u_{1}+\left[r_{1} v_{x}^{*}(t) v_{z}+r_{2} \theta+r_{3} u_{2}+r_{4} e(\dot{\theta}+\dot{\psi})-r_{4} d \sin \psi\right] \dot{\theta} } \\
& -r_{4} e(\dot{\theta}+\dot{\psi})^{2} .
\end{aligned}
$$

Clearly, the feedback laws

$$
\begin{aligned}
& u_{1}=-l_{1}\left[r_{1} v_{z}-r_{4} c(\dot{\theta}+\dot{\psi}) \cos \psi\right], \\
& \left.u_{2}=-l_{2} \dot{\theta}-\left[\frac{r_{1}}{r_{3}} v_{x}^{*}(t) v_{z}+\frac{r_{2}}{r_{3}} \theta\right]-\frac{r_{4}}{r_{3}}[e(\dot{\theta}+\dot{\psi})-d \sin \psi)\right],
\end{aligned}
$$

where $l_{1}$ and $l_{2}$ are positive constants, yield

$$
\dot{V}=-l_{1}\left[r_{1} v_{z}-r_{4} c(\dot{\theta}+\dot{\psi}) \cos \psi\right]^{2}-l_{2} \dot{\theta}^{2}-r_{4} e(\dot{\theta}+\dot{\psi})^{2},
$$

which satisfies $\dot{V} \leq 0$ in $D$. Using LaSalle's principle, it is easy to prove asymptotic stability of the origin of the closed loop defined by the equations (37)-(40) and the feedback control laws (45)-(46). Note that the positive gains $r_{i}, i=1,2,3,4$ and $l_{j}, j=1,2$, can be chosen arbitrarily to achieve good closed loop responses.

\section{Simulations}

The feedback control laws developed in the previous sections are implemented here for a spacecraft. The physical parameters used in the simulations are $m=600 \mathrm{~kg}, I=720 \mathrm{~kg} / \mathrm{m}^{2}$, $m_{f}=100 \mathrm{~kg}, I_{f}=90 \mathrm{~kg} / \mathrm{m}^{2}, a=0.2 \mathrm{~m}, b=0.3 \mathrm{~m}, p=0.2 \mathrm{~m}, F=2300 \mathrm{~N}$ and $\epsilon=0.19 \mathrm{~kg} \cdot \mathrm{m}^{2} / \mathrm{s}$.

We consider stabilization of the spacecraft in orbital transfer, suppressing the transverse and pitching motion of the spacecraft and sloshing of fuel while the spacecraft is accelerating. In other words, the control objective is to stabilize the relative equilibrium corresponding to a constant axial spacecraft acceleration of $3.286 \mathrm{~m} / \mathrm{s}^{2}$ and $v_{z}=\theta=\dot{\theta}=\psi=\dot{\psi}=0$. 


\subsection{Linearization-based controller}

In this section, an LQR controller of the form (31) is applied to the complete nonlinear system (11)-(14). Using the physical parameters given above, the A and B matrices defined by equation (30) were computed as

$$
\begin{aligned}
& \mathbf{A}=\left[\begin{array}{cccc}
0 & 1 & 0 & 0 \\
0 & 0 & -0.005 & 0.0002 \\
0 & 0 & 0 & 1 \\
0 & 0 & -0.6987 & -0.0023
\end{array}\right] \\
& \mathbf{B}=\left[\begin{array}{cc}
0 & 0 \\
0.7629 & 0.0014 \\
0 & 0 \\
-1.4243 & -0.0013
\end{array}\right]
\end{aligned}
$$

Choosing the weighting matrices as

$$
\begin{aligned}
& \mathbf{Q}=\left[\begin{array}{llll}
1 & 0 & 0 & 0 \\
0 & 1 & 0 & 0 \\
0 & 0 & 1 & 0 \\
0 & 0 & 0 & 1
\end{array}\right] \\
& \mathbf{R}=\left[\begin{array}{cc}
10 & 0 \\
0 & 0.01
\end{array}\right]
\end{aligned}
$$

the LQR gain matrix for the linear system (29) was found as

$$
\mathbf{K}=\left[\begin{array}{cccc}
0.3153 & 1.2214 & -0.3496 & -0.2696 \\
0.7627 & 2.8027 & 0.6462 & 0.0764
\end{array}\right]
$$

This gain matrix yields the following eigenvalues for the closed-loop system matrix $\mathbf{A}-\mathbf{B K}$ : $(-0.3312 \pm 0.8233 i,-0.3297 \pm 0.3294 i)$.

Time responses shown in Fig. 4 and Fig. 5 correspond to the initial conditions $v_{x 0}=10000 \mathrm{~m} / \mathrm{s}$, $v_{z 0}=0, \theta_{0}=2^{\circ}, \dot{\theta}_{0}=0.57^{\circ} / s, \psi_{0}=15^{\circ}$, and $\dot{\psi}_{0}=0$. As can be seen in the figures, the LQR controller stabilizes the pitch and slosh dynamics, but fails to stabilize the transverse velocity to zero. The controller results in a steady-state error of $v_{z}=-349.1 \mathrm{~m} / \mathrm{s}$.

\subsection{Lyapunov-based controller}

In this section, we demonstrate the effectiveness of the Lyapunov-based controller (45)-(46) by applying to the complete nonlinear system (11)-(14).

Time responses shown in Fig. 6 and Fig. 7 correspond to the initial conditions $v_{x 0}=10000 \mathrm{~m} / \mathrm{s}$, $v_{z 0}=350 \mathrm{~m} / \mathrm{s}, \theta_{0}=2^{\circ}, \dot{\theta}_{0}=0.57^{\circ} / \mathrm{s}, \psi_{0}=30^{\circ}$, and $\dot{\psi}_{0}=0$. As can be seen in the figures, the transverse velocity, attitude angle and the slosh angle converge to the relative equilibrium at zero while the axial velocity $v_{x}$ increases and $\dot{v}_{x}$ tends asymptotically to $3.286 \mathrm{~m} / \mathrm{s}^{2}$. Note that there is a trade-off between good responses for the directly actuated degrees of freedom (the transverse and pitch dynamics) and good responses for the unactuated degree of freedom (the slosh dynamics); the controller given by (45)-(46) with parameters $r_{1}=10^{-7}, r_{2}=10, r_{3}=$ $10^{2}, r_{4}=10^{-2}, l_{1}=10^{3}, l_{2}=1$ represents one example of this balance. 

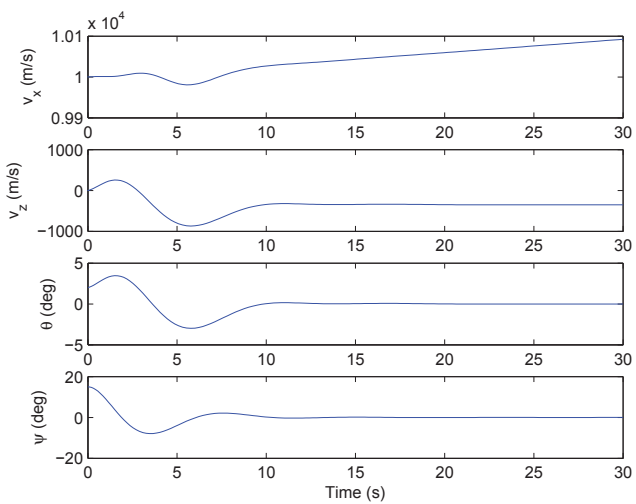

Fig. 4. Time responses of state variables $v_{x}, v_{z}, \theta$, and $\psi$ (LQR controller).
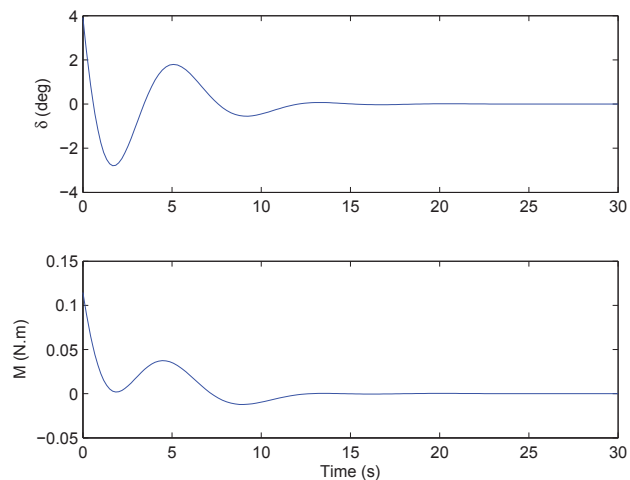

Fig. 5. Gimbal deflection angle $\delta$ and pitching moment $M$ (LQR controller).
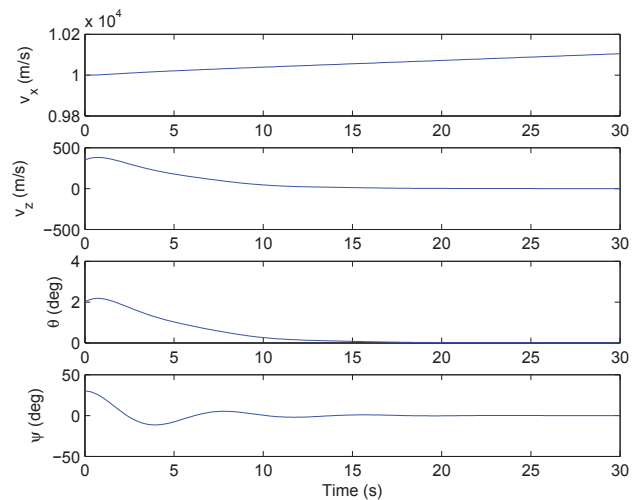

Fig. 6. Time responses of state variables $v_{x}, v_{z}, \theta$, and $\psi$ (Lyapunov-based controller). 

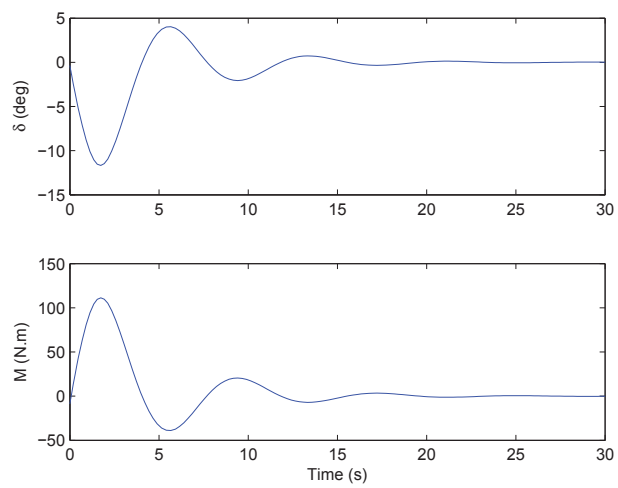

Fig. 7. Gimbal deflection angle $\delta$ and pitching moment $M$ (Lyapunov-based controller).

\section{Conclusions}

We have shown that a linear controller, while successful in stabilizing the pitch and slosh dynamics, fails to control the transverse dynamics of a spacecraft. We have designed a Lyapunov-based nonlinear feedback control law that achieves stabilization of the pitch and transverse dynamics as well as suppression of the slosh mode, while the spacecraft accelerates in the axial direction. The effectiveness of this control feedback law has been illustrated through a simulation example.

The many avenues considered for future research include problems involving higher-frequency slosh modes, multiple propellant tanks, and three dimensional maneuvers. Future research also includes designing nonlinear control laws that achieve robustness, insensitivity to system and control parameters, and improved disturbance rejection. In particular, we plan to explore the use of sliding mode controllers to accomplish this.

\section{References}

Adler, J. M., Lee, M. S. \& Saugen, J. D. (1991). Adaptive control of propellant slosh for launch vehicles, SPIE Sensors and Sensor Integration 1480: 11-22.

Biswal, K. C., Bhattacharyya, S. K. \& Sinha, P. K. (2004). Dynamic characteristics of liquid filled rectangular tank with baffles, IE (I) Journal-CV 84: 145-148.

Blackburn, T. R. \& Vaughan, D. R. (1971). Application of linear optimal control and filtering theory to the saturn v launch vehicle, IEEE Transactions on Automatic Control 16(6): 799-806.

Bryson, A. E. (1994). Control of Spacecraft and Aircraft, Princeton University Press.

Cho, S., McClamroch, N. H. \& Reyhanoglu, M. (2000a). Dynamics of multibody vehicles and their formulation as nonlinear control systems, Proceedings of American Control Conference, pp. 3908-3912.

Cho, S., McClamroch, N. H. \& Reyhanoglu, M. (2000b). Feedback control of a space vehicle with unactuated fuel slosh dynamics, Proceedings of AIAA Guidance, Navigation, and Control Conference, pp. 354-359.

Feddema, J. T., Dohrmann, C. R., Parker, G. G., Robinett, R. D., Romero, V. J. \& Schmitt, D. J. (1997). Control for slosh-free motion of an open container, IEEE Control Systems 
Magazine pp. 29-36.

Freudenberg, J. \& Morton, B. (1992). Robust control of a booster vehicle using $h_{\infty}$ and ssv techniques, Proceedings of the 31st IEEE Conference on Decision and Control, pp. 2448-2453.

Grundelius, M. (2000). Iterative optimal control of liquid slosh in an industrial packaging machine, Proceedings of the 39th IEEE Conference on Decision and Control, pp. 3427-3432.

Grundelius, M. \& Bernhardsson, B. (1999). Control of liquid slosh in an industrial packaging machine, Proceedings of IEEE International Conference on Control Applications, pp. 1654-1659.

Hubert, C. (2003). Behavior of Spinning Space Vehicles with Onboard Liquids, NASA/KSC Contract NAS10-02016.

Hubert, C. (2004). Design and flight performance of a system for rapid attitude maneuvers by a spinning vehicle, Proceedings of the 27th Annual AAS Guidance and Control Conference.

Meirovitch, L. \& Kwak, M. K. (1989). State equations for a spacecraft with maneuvering flexible appendages in terms of quasi-coordinates, Applied Mechanics Reviews 42(11): 161-170.

Peterson, L. D., Crawley, E. F. \& Hansman, R. J. (1989). Nonlinear fluid slosh coupled to the dynamics of a spacecraft, AIAA Journal 27(9): 1230-1240.

Reyhanoglu, M. (2003). Maneuvering control problems for a spacecraft with unactuated fuel slosh dynamics, Proceedings of IEEE Conference on Control Applications, pp. 695-699.

Reyhanoglu, M., Cho, S. \& McClamroch, N. H. (2000). Discontinuous feedback control of a special class of underactuated mechanical systems, International Journal of Robust and Nonlinear Control 10(4): 265-281.

Reyhanoglu, M., van der Schaft, A. J., McClamroch, N. \& Kolmanovsky, I. (1996). Nonlinear control of a class of underactuated systems, Proceedings of IEEE Conference on Decision and Control, pp. 1682-1687.

Reyhanoglu, M., van der Schaft, A. J., McClamroch, N. \& Kolmanovsky, I. (1999). Dynamics and control of a class of underactuated mechanical systems, IEEE Transactions on Automatic Control 44(9): 1663-1671.

Sidi, M. J. (1997). Spacecraft Dynamics and Control, Cambridge University Press, New York.

Terashima, K. \& Schmidt, G. (1994). Motion control of a cart-based container considering suppression of liquid oscillations, Proceedings of IEEE International Symposium on Industrial Electronics, pp. 275-280.

Venugopal, R. \& Bernstein, D. S. (1996). State space modeling and active control of slosh, Proceedings of IEEE International Conference on Control Applications, pp. 1072-1077.

Wie, B. (2008). Space Vehicle Dynamics and Control, AIAA Education Series.

Yano, K., Higashikawa, S. \& Terashima, K. (2001). Liquid container transfer control on 3d transfer path by hybrid shaped approach, Proceedings of IEEE International Conference on Control Applications, pp. 1168-1173.

Yano, K. \& Terashima, K. (2001). Robust liquid container transfer control for complete sloshing suppression, IEEE Transactions on Control Systems Technology 9(3): 483-493.

Yano, K., Toda, T. \& Terashima, K. (2001). Sloshing suppression control of automatic pouring robot by hybrid shape approach, Proceedings of the 40th IEEE Conference on Decision and Control, pp. 1328-1333. 


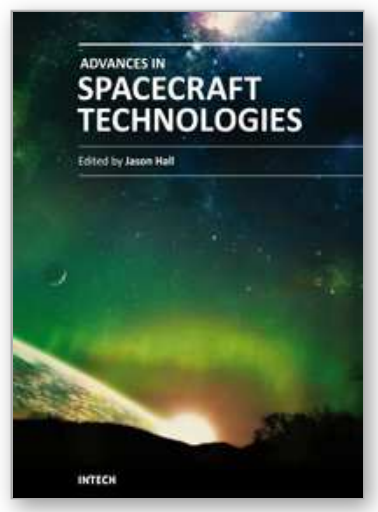

\author{
Advances in Spacecraft Technologies \\ Edited by Dr Jason Hall
}

ISBN 978-953-307-551-8

Hard cover, 596 pages

Publisher InTech

Published online 14, February, 2011

Published in print edition February, 2011

The development and launch of the first artificial satellite Sputnik more than five decades ago propelled both the scientific and engineering communities to new heights as they worked together to develop novel solutions to the challenges of spacecraft system design. This symbiotic relationship has brought significant technological advances that have enabled the design of systems that can withstand the rigors of space while providing valuable space-based services. With its 26 chapters divided into three sections, this book brings together critical contributions from renowned international researchers to provide an outstanding survey of recent advances in spacecraft technologies. The first section includes nine chapters that focus on innovative hardware technologies while the next section is comprised of seven chapters that center on cutting-edge state estimation techniques. The final section contains eleven chapters that present a series of novel control methods for spacecraft orbit and attitude control.

\title{
How to reference
}

In order to correctly reference this scholarly work, feel free to copy and paste the following:

Mahmut Reyhanoglu (2011). Modelling and Control of Space Vehicles with Fuel Slosh Dynamics, Advances in Spacecraft Technologies, Dr Jason Hall (Ed.), ISBN: 978-953-307-551-8, InTech, Available from: http://www.intechopen.com/books/advances-in-spacecraft-technologies/modelling-and-control-of-spacevehicles-with-fuel-slosh-dynamics

\section{INTECH}

open science | open minds

\section{InTech Europe}

University Campus STeP Ri

Slavka Krautzeka 83/A

51000 Rijeka, Croatia

Phone: +385 (51) 770447

Fax: +385 (51) 686166

www.intechopen.com

\section{InTech China}

Unit 405, Office Block, Hotel Equatorial Shanghai

No.65, Yan An Road (West), Shanghai, 200040, China

中国上海市延安西路65号上海国际贵都大饭店办公楼 405 单元

Phone: +86-21-62489820

Fax: $+86-21-62489821$ 
(C) 2011 The Author(s). Licensee IntechOpen. This chapter is distributed under the terms of the Creative Commons Attribution-NonCommercialShareAlike-3.0 License, which permits use, distribution and reproduction for non-commercial purposes, provided the original is properly cited and derivative works building on this content are distributed under the same license. 